\title{
Re-entrance of the Metallic Conductance in a Mesoscopic Proximity Superconductor
}

\author{
P. Charlat ${ }^{1}$, H. Courtois ${ }^{1}$, Ph. Gandit ${ }^{1}$, D. Mailly ${ }^{2}$, A.F. Volkov ${ }^{3}$, and B. Pannetier ${ }^{1}$ \\ ${ }^{1}$ Centre de Recherches sur les Très Basses Températures-C.N.R.S. associé à l'Université Joseph Fourier, 25 Av. des Martyrs, \\ 38042 Grenoble, France \\ ${ }^{2}$ Laboratoire de Microstructures et de Microélectronique-C.N.R.S., 196 Av. H. Ravera, 92220 Bagneux, France \\ ${ }^{3}$ Institute of Radio Engineering and Electronics, Russian Academy of Sciences, Mokhovaya St. 11, 103907 Moscow, Russia
}

We present an experimental study of the diffusive transport in a normal metal near a superconducting interface, showing the re-entrance of the metallic conductance at very low temperature. This new mesoscopic regime comes in when the thermal coherence length of the electron pairs exceeds the sample size. This re-entrance is suppressed by a bias voltage given by the Thouless energy and can be strongly enhanced by an Aharonov Bohm flux. Experimental results are well described by the linearized quasiclassical theory.

During the last few years, the proximity effect between a superconductor $(\mathrm{S})$ and a normal $(\mathrm{N})$ metal has met a noticeable revival, thanks to spectacular progress in the fabrication of samples of mesoscopic size [1]. Experimental study of the transport near a $\mathrm{S}-\mathrm{N}$ interface has shown that the proximity effect strongly affects electron transport in mesoscopic S-N systems : the deviation $\Delta G$ of the conductance from its normal-state value strongly depends on temperature $T$ and oscillates in an applied magnetic field $H$ if a $\mathrm{N}$ loop is present [2 4]. Various theoretical approaches were suggested to explain this behavior. A scattering matrix method based upon Landauer formula [5] as well as a numerical solution of the Bogolubov-de Gennes equations [6] were used. These studies demonstrated that superconductivity does not affect the charge transfer in the $\mathrm{N}$ metal if the temperature $T$ and the voltage $V$ are zero, i.e. $\Delta G$ is zero at zero energy. A more powerful method based on the equations for the quasiclassical Green's functions [10 was used to obtain the dependence of $\Delta G$ on $T$ and $V$. It has been established [9] that at $V=0$ the deviation of the conductance $\Delta G$ increases from zero at $T=0$ (if electronelectron interaction in $\mathrm{N}$ is negligeable) with increasing $T$, reaches a maximum at approximately the Thouless temperature $\epsilon_{c} / k_{B}=\hbar D / k_{B} L^{2}$ and decreases to zero at $T>>\epsilon_{c} / k_{B}$. This constitutes the re-entrance effect for the metallic conductance of the N metal. Similar dependence of $\Delta G(V)$ at $T=0$ has been found in [10] both in a numerical solution of the Bogoliubov-de Gennes equations and in a analytical solution of the equations for the quasiclassical Green's functions.

The physics behind this re-entrance effect involves non equilibrium effects between quasiparticles injected by the $\mathrm{N}$ reservoirs and electron pairs leaking from $\mathrm{S}$. At the N-S interface, an incident electron is reflected into a hole of the same energy $\epsilon$ compared to the Fermi level $E_{F}$, but with a slight change in wave-vector $\delta k$ due to the branch crossing : $\delta k / k_{F}=\epsilon / E_{F}, k_{F}$ being the Fermi wavevector. The phase conjugation between the electron and the hole results in a finite pair amplitude involving states $\left(k_{F}+\epsilon / \hbar v_{F},-k_{F}+\epsilon / \hbar v_{F}\right)$, $v_{F}$ being the Fermi velocity. Such a pair maintains coherence in $\mathrm{N}$ up to the energy-dependent diffusion length $L_{\epsilon}=\sqrt{\hbar D / \epsilon}$, 17, 11] which coincides with the well-known thermal length $L_{T}=\sqrt{\hbar D / k_{B} T}$ at $\epsilon=2 \pi k_{B} T$.

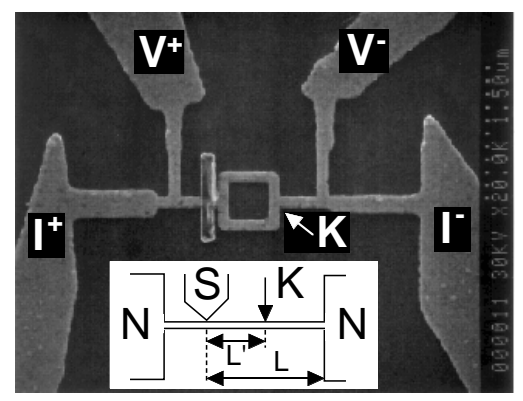

FIG. 1. Micrograph of the sample made of a continuous $\mathrm{Cu}(=\mathrm{N})$ loop in contact with a single $\mathrm{Al}(=\mathrm{S})$ island. Inset : simple model. At half integer magnetic flux, a condition $F=0$ is enforced at the point $\mathrm{K}$ at $L^{\prime}=1 \mu \mathrm{m}$ from $\mathrm{S}$. The distances between $\mathrm{S}$ and the right and left $\mathrm{N}$ reservoirs are respectively $L=2 \mu \mathrm{m}$ and $0.5 \mu \mathrm{m}$.

In the high-temperature regime $L_{T}<L$ or equivalently $\epsilon_{c}<k_{B} T$, it is well-known that proximity effect results in the subtraction of a length $L_{T}$ of $\mathrm{N}$ metal from the resistance of a S-N junction. In the low temperature $L_{T}>L$ or $\epsilon_{c}>k_{B} T$ and low voltage $e V<\epsilon_{c}$, electron pairs are coherent over the whole sample. The proximity effect on the $\mathrm{N}$ metal resistance is still predicted to be zero. In this Letter, we report the experimental realization of both limits $\left(L<L_{T}\right.$ and $\left.L>L_{T}\right)$ and the observation of the re-entrance of the metallic conductance in a mesoscopic proximity superconductor. The low-temperature re-entrant regime is destroyed by increasing the temperature [9] or the voltage [10]. As will be discussed below, an Aharonov-Bohm flux modifies the effective length of the sample and therefore shifts the energy crossover of the re-entrant regime.

Fig. 11 shows a micrograph of the sample made of a square copper $(\mathrm{Cu})$ loop in contact with a single aluminum (Al) island. The loop, although not essential for 
the occurrence of the re-entrance effect allows one to control boundary conditions for the pair amplitude. The $\mathrm{Cu}$ wire width is $150 \mathrm{~nm}$ and its thickness is about $40 \mathrm{~nm}$. The distance between the $\mathrm{Cu}$ loop and the $\mathrm{Al}$ island is about $100 \mathrm{~nm}$, whereas the perimeter of the loop is $2 \mu \mathrm{m}$. One should note that the sample geometry differs from all previous sample geometries with two superconducting contacts 24 in that there is a single superconducting phase and therefore no possible Josephson contribution. Two voltage probes measure the distribution at the outflows of the reservoirs, which are the wide contact pads at both ends of the $\mathrm{Cu}$ wire. The $\mathrm{Cu}$ surface is in-situ cleaned before $\mathrm{Al}$ deposition in order to ensure an optimum transparency of the $\mathrm{Cu} / \mathrm{Al}$ interface [4].

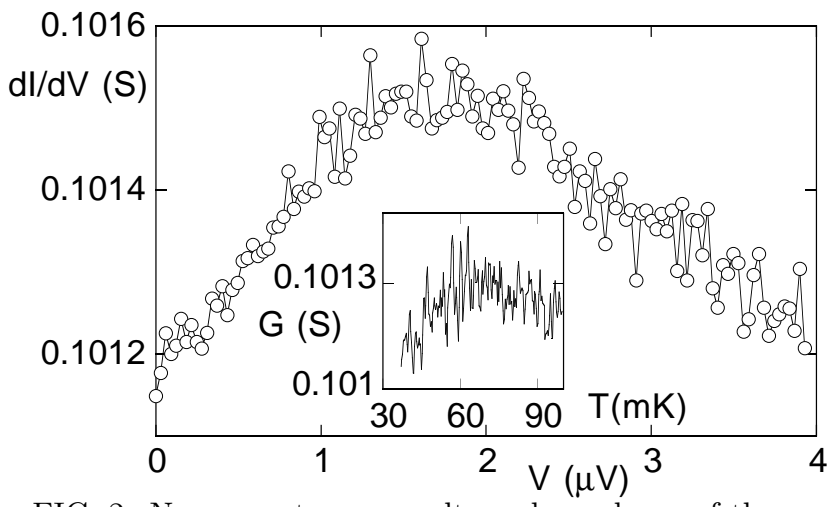

FIG. 2. Non monotoneous voltage dependence of the sample conductance at $T=22 \mathrm{mK}$; the normal-state conductance $G_{N}$ is $0.0937 \mathrm{~S}$. Inset: Temperature dependence. Measurement current of $70 \mathrm{nA}$ in both curves.

We performed transport measurements in a $\mu$-metalshielded dilution refrigerator down to $20 \mathrm{mK}$. From the normal-state conductance $G_{N}=0.0937 \mathrm{~S}$ we find a diffusion coefficient $D=70 \mathrm{~cm}^{2} / \mathrm{s}$, an elastic mean free path $l_{e}=13 \mathrm{~nm}$ and a thermal coherence length $L_{T}=92$ $\mathrm{nm} / \sqrt{T}$. Aluminum islands become superconducting below $T_{c} \simeq 1.4 \mathrm{~K}$. The behaviour of the conductance in the high-temperature regime $L_{T}<L$ (i.e. above $500 \mathrm{mK}$ ) is very similar to the two-island case [4. At lower temperatures, so that $L_{T} \simeq L$, and zero magnetic field, we observe a decrease of the low-voltage conductance (Fig. 2 inset). This occurs below $50 \mathrm{mK}$, at the temperature where a Josephson coupling would be expected in a twoislands geometry. The voltage dependence of the measured conductance shows the most striking behaviour i.e. an increase of the conductance when the bias voltage is increased (Fig. 2). This non-linear behaviour discards an interpretation in terms of weak localization, which is known to be insensitive to voltage. The conductance peak is observed at a bias voltage (about $1.7 \mu \mathrm{V}$ ) of the order of the calculated Thouless energy $\epsilon_{c}=1.1 \mu \mathrm{V}$ related with a sample length $L=2 \mu \mathrm{m}$. In Fig. 2 inset, the peak position is also consistent with the Thouless temperature $\epsilon_{c} / k_{B}=13 \mathrm{mK}$. One can note that the dis- cussed energies are much smaller than Al energy gap $\Delta$.

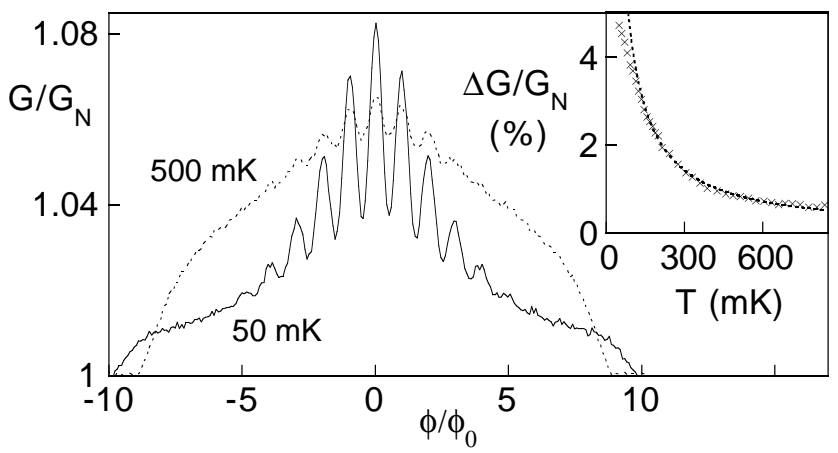

FIG. 3. Magnetoconductance oscillations showing $\Phi_{0}=h / 2 e$ flux-periodicity at $T=50 \mathrm{mK}$ (solid line) and at $T=500 \mathrm{mK}$ (dashed). Re-entrance of the resistance is visible at half integer magnetic flux and at high field. Inset : oscillations amplitude together with a $1 / \mathrm{T}$ power law fit.

Let us now analyze the effect of the magnetic field. Fig. 3 shows oscillations of the magnetoconductance with a periodicity of one flux quantum $\phi_{0}=h / 2 e$ in the loop area. Here the re-entrance effect can be very clearly seen at $\phi=\phi_{0} / 2,3 \phi_{0} / 2$ and at higher field. As previously observed in two-island samples, the oscillation amplitude decays slowly with a $1 / T$-power-law down to $200 \mathrm{mK}$ (Fig. 3 inset). Fig. 4 shows the temperature dependence of the conductance for various values of the magnetic flux in the loop. On this scale, the re-entrance at zero-field is hardly distinguishable [12]. At $\phi=\phi_{0} / 2$, the conductance maximum is near $500 \mathrm{mK}$ and the re-entrance has a much larger amplitude. At $\phi=\phi_{0}$, the curve is close to the zero-field case, and at $\phi=3 \phi_{0} / 2$, close to the $\phi_{0} / 2$ case. Increasing further the magnetic field (Fig. 4 left part), the conductance peak is displaced to higher temperature and the $\phi_{0}$-periodic modulation is suppressed. Fig. 5 shows the conductance peaks energies obtained from both the current-voltage characteristics and temperature dependence of the conductance as a function of the magnetic flux. Hence the magnetic field has two effects on the re-entrance : (i) a large $\phi_{0}$ oscillation of the peak position at low field ; (ii) a monotonous shift at higher fields.

Most of our observations can be analysed in the framework of the quasiclassical theory for inhomogenous superconductors [7,9,11. We present here a simplified version that however keeps the essential physical features. We consider the mesoscopic regime where the inelastic scattering length is larger than the sample length $L$ between the reservoirs. The flow of electrons at a particular energy $\epsilon$ is then uniform over the sample, and one has to consider transport through independent channels at the energy $\epsilon$. In a perfect reservoir, electrons follow the Fermi equilibrium distribution at the temperature $T$ and chemical potential $\mu$. Charges are injected in the system from one reservoir at $\mu=e V$ and transferred to the other, so 
that the current is carried by electrons within an energy window $[0, \mu=e V]$ with a thermal broadening $k_{B} T$. Let us assume that proximity effect can be accounted for as a conductivity enhancement $\delta \sigma(\epsilon, x)$ depending on both the energy $\epsilon$ and the distance $x$ from the $\mathrm{S}$ interface. From the behaviour of $\delta \sigma(\epsilon, x)$ it is then straightforward to calculate the excess conductance $\delta g(\epsilon)$ for the precise geometry of the sample. The excess conductance $\delta G(V, T)$ at voltage $\mathrm{V}$ and temperature $\mathrm{T}$ writes :

$$
\delta G(V, T)=\int_{-\infty}^{\infty} \delta g(\epsilon) P(V-\epsilon) d \epsilon
$$

where $P(\epsilon)=\left[4 k_{B} T \cosh ^{2}\left(\epsilon / 2 k_{B} T\right)\right]^{-1}$ is a thermal kernel which reduces to the Dirac function at $T=0$. Hence, the low-temperature differential conductance $d I / d V=$ $G_{N}+\delta G$ probes the proximity-induced excess conductance $\delta g(\epsilon)$ at energy $\epsilon=e V$ with a thermal broadening $k_{B} T$. Independent measurements of the excess conductance as a function of $T$ and $V$ agree with Eq. 1 for not too low temperatures and voltages, provided the chemical potential in the reservoirs is taken as equal to the measured voltage times a geometrical factor of 1.05. This correspondence however fails for energies less than about $5 \mu \mathrm{eV}$ : we believe that at these energies the inelastic collision rate is too small to ensure a good thermalisation in the reservoirs [13].

In $\mathrm{N}$ and at zero magnetic field, $F(\epsilon, x)$ follows the Usadel equation that for small $F$ can be linearized as :

$$
\hbar D \partial_{x}^{2} F+\left(2 i \epsilon-\frac{\hbar D}{L_{\varphi}^{2}}\right) F=0
$$

The linearization used above is a very crude approximation, since near the interface which is believed to be clean, the pair amplitude should be large (in this approximation, we have $F(\epsilon \ll \Delta, 0)=-i \pi / 2$ for a perfectly transparent interface [13]). At the contact with a $\mathrm{N}$ reservoir $F$ is assumed to be zero. However, this simple formulation enables a straightforward understanding of the physical root of the conductance enhancement in a proximity system. Indeed Eq. 2 features simply a diffusion equation for the pair amplitude $F$ at the energy $\epsilon$ with a decay length $L_{\epsilon}$ and a cut-off at $L_{\varphi}$. At a particular energy $\epsilon$, the real part of the pair amplitude is zero at the $\mathrm{S}$ interface, maximum at a distance $L_{\epsilon}$ if $L_{\epsilon} \ll L_{\varphi}, L$ and then decays in an oscillating way. The pair amplitude $F$ is responsible for the local enhancement of the conductivity $\delta \sigma(\epsilon, x)=\sigma_{N}(\operatorname{Re}[F(\epsilon, x)])^{2}$ for small $F, \sigma_{N}$ being the normal-state conductivity [7.9611. It involves two contributions : a positive and dominant one which is similar to the Maki-Thompson fluctuation term in superconductors above $T_{c}$ [14] and a negative one related to the decrease of the density of states. The two contributions cancel each other at zero energy [15].

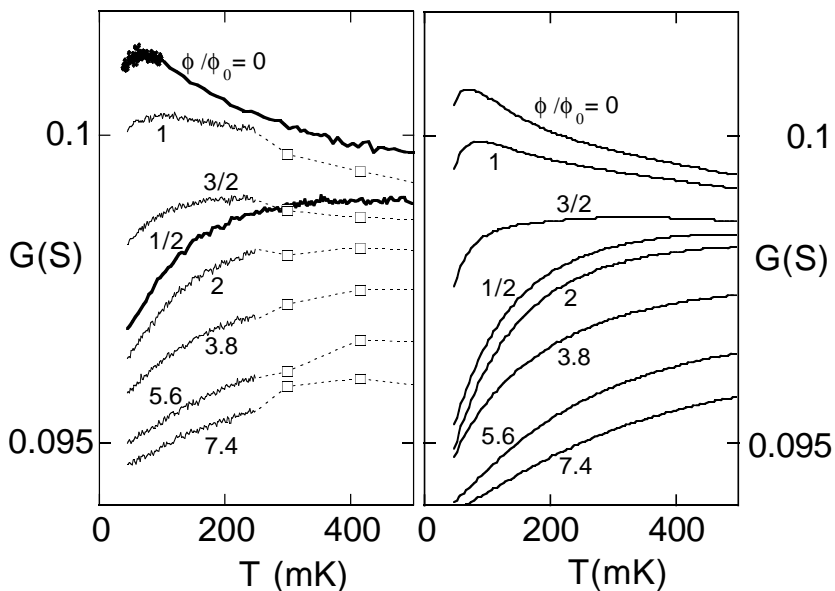

FIG. 4. Left : Measured temperature dependence of the conductance at different values of the magnetic flux $\Phi$ in units of the flux-quantum : $\Phi / \Phi_{0}=0 ; 1 / 2 ; 1 ; 3 / 2 ; 2 ; 3.8 ; 5.6 ; 7.4$. Measurement current is $200 \mathrm{nA}$. Right: Calculated conductance in the linear approximation for the sample model of Fig. 1 inset and at the same values of the flux. The only adjustable parameter is the effective width of the wires.

We modelize the sample as two independent S-N circuits in series as shown in the inset of Fig. 1. This is our main approximation. It describes the main physics of our particular geometry and illustrates more general situations. Both circuits consist of a $\mathrm{N}$-wire between a superconductor $\mathrm{S}$ and a normal reservoir N. Along the wire, the excess conductivity $\delta \sigma(\epsilon, x)$ at a given energy $\epsilon$ increases from zero at the N-S interface to a maximum of about $0.3 \sigma_{N}$ at a distance $L_{\epsilon}$ from the interface (if $\left.L \ll L_{\varphi}\right)$ and then decays exponentially with $x$. The integrated excess conductance $\delta g(\epsilon)$ of the whole sample rises from zero with an $\epsilon^{2}$ law at low energy, reaches a maximum of $0.15 G_{N}$ at about $5 \epsilon_{c}$ and goes back to zero at higher energy with a $1 / \sqrt{\epsilon}$ law. This behaviour is indeed confirmed in the experimental results in Fig.2. We observe a conductance peak as function of both temperature and voltage. The conductance is maximum for a temperature $(50 \mathrm{mK})$ close to the calculated crossover temperature $\left(5 \epsilon_{c} / k_{B}=65 \mathrm{mK}\right)$, see Fig. 2 inset. Only qualitative agreement between the observed $(1.7 \mu \mathrm{V})$ and calculated energy $\left(5 \epsilon_{c}=5.5 \mu V\right)$ is obtained. This discrepancy is believed to be due to insufficient energy relaxation efficiency at low energy in the $\mathrm{Cu}$ reservoirs.

Because of the loop geometry, a magnetic field induces an Aharonov-Bohm flux, which changes the boundary conditions on the pair amplitude $F(\epsilon)$. At zero magnetic flux, $F(\epsilon)$ is zero only at the contact with the normal reservoirs. At half magnetic flux, destructive interference of the pair functions in the two branches enforces a zero in $F(\epsilon)$ at the node $\mathrm{K}$ (see Fig. 1 inset). Consequently, the pair amplitude is also zero between the loop and the $\mathrm{N}$ reservoir. Half a flux-quantum then reduces the effective sample size to the length $L^{\prime}$ between the $\mathrm{S}$ interface and the point $\mathrm{K}$. In the intermediate temperature regime 
$k_{B} T>\epsilon_{c}$ (or $\left.L_{T}<L\right)$, this modulates the conductance with a relative amplitude of the order of $\epsilon_{c} / k_{B} T$ [13], in qualitative agreement with the experiment.

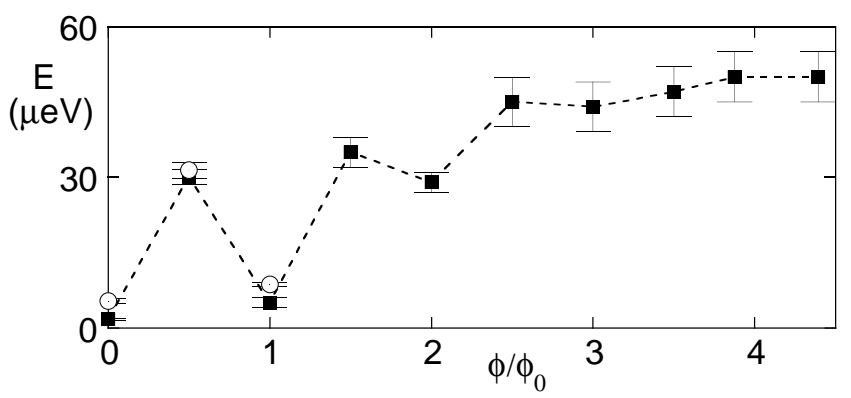

FIG. 5. Energy of the conductance maximum as a function of the magnetic flux $\phi$ in units of $\Phi_{0}$. The black square dots are obtained from the voltage-conductance $d I / d V(V)$ characteristics at $T=100 \mathrm{mK}$, except for $\Phi / \Phi_{0}=0$ and $1(T=22$ and $45 \mathrm{mK}$ ). The white circles are obtained from Fig. 4 . The discrepancies inbetween reflect the imperfections of the reservoirs at low energies.

As an additional effect of the magnetic field $H$, the phase-memory length $L_{\varphi}$ is renormalized due to the finite width $w$ of the $\mathrm{Cu}$ wire [16] :

$$
L_{\varphi}^{-2}(H)=L_{\varphi}^{-2}(0)+\frac{\pi^{2}}{3} \frac{H^{2} w^{2}}{\Phi_{0}^{2}}
$$

When smaller than the sample length $\mathrm{L}$, the phasememory length $L_{\varphi}(H)$ plays the role of an effective length for the sample. As a result, the conductance peak is shifted to higher temperatures and energies when the magnetic field is increased, see Fig.4 and 5. At high magnetic field, the position of the conductance maxima does not increase as rapidly as could be expected because of the field-induced depletion of the gap $\Delta$. In the right part of Fig. 4, we show the calculated conductance using of Eq. 1-3 in the modelized geometry of Fig.1 inset in the case of a fully transparent interface. The only free parameter is the width of the wires which has been adjusted so that the experimental damping of the amplitude of the magnetoconductance oscillations by the magnetic field, see Fig. 3, is well described by the calculation. The discrepancy between the fitted value $w=65$ $\mathrm{nm}$ and the measured value is attributed to deviations of sample geometry from our simple model. Our calculation accounts for both the global shape and amplitude of the curves and for their behaviour as a function of the magnetic flux. This is particularly remarkable in respect with the strong assumptions of the model. One should note that qualitative shape and amplitude of the curves are conserved if non-linearized Usadel equations or slightly different geometrical parameters are used.

In conclusion, we have measured the energy dependence of the proximity effect on the conductance near an N-S junction. As predicted in recent works [9,10, we have observed the re-entrance of the metallic conductance when all energies involved are below the Thouless energy of the sample. In contrast with a very recent similar observation [17], the energy crossover has been tracked as a function of temperature, voltage and magnetic field. Our experimental results are well described by the linearized Usadel equations from the quasiclassical theory.

We thank P. Butaud, M. Dévoret, D. Estève, B. Spivak, T. Stoof, A. Zaikin, and F. Zhou for stimulating discussions. A. F. V. thanks P. Monceau for hospitality, the Russian Fund for Fundamental Research and the collaboration program between the Ecole Normale Superieure de Paris and the Landau Institute for Theoritical Physics for support. We also acknowledge financial support from Région Rhône-Alpes and D.R.E.T..

[1] See references in "Mesoscopic Superconductivity", ed. by F. Hekking, G. Schön, and D. Averin, Physica B 203, (1994).

[2] V.T. Petrashov, V.N. Antonov, P. Delsing, and T. Claeson, Phys. Rev. Lett. 70, 347 (1993); Phys. Rev. Lett. 74, 5268 (1995).

[3] A. Dimoulas, J.P. Heida, B.J. van Wees, T.M. Klapwijk, W.v.d. Graaf, and G. Borghs, Phys. Rev. Lett. 74, 602 (1995).

[4] H. Courtois, Ph. Gandit, D. Mailly, and B. Pannetier Phys. Rev. Lett. 76, 130 (1996).

[5] C. W. J. Beenakker, Phys. Rev. B 46, 12841 (1992).

[6] C. J. Lambert, J. Phys. Condens. Matter 3, 6579 (1991).

[7] A. F. Volkov, A.V. Zaitsev, and T. M. Klapwijk, Physica C 210, 21 (1993); A. F. Volkov and A.V. Zaitsev, Phys. Rev. B 53, 9267 (1996); A. V. Zaitsev, JETP Lett. 51, 35 (1990) and Phys. Lett. 194, 315 (1994).

[8] A. A. Golubov, F. Wilhelm and A. D. Zaikin, to appear in Phys. Rev. B.

[9] Y. V. Nazarov and T. H. Stoof, Phys. Rev. Lett. 76, 823 (1996).

[10] A.F. Volkov, N. Allsopp and C.J. Lambert, J. Phys. Condens. Matter 8, L45 (1996).

[11] F. Zhou, B. Spivak, and A. Zyuzin, Phys. Rev. B 52, 4467 (1995).

[12] We observed recently a re-entrance effect with a much larger amplitude in smaller samples, see P. Charlat, $\mathrm{H}$. Courtois, Ph. Gandit, D. Mailly, A. Volkov, and B. Pannetier, to appear in Proceedings of the LT21 Conference, Czech. J. of Phys. 46, S6 (1996).

[13] P. Charlat, H. Courtois, Ph. Gandit, D. Mailly, and B. Pannetier, unpublished.

[14] B.R. Patton, Proc. 13th Int. Conf. on Low Temp. Phys., 1972, Ed. by W.S. O'Sullivan, Plenum (New York).

[15] A. F. Volkov and V. V. Pavlovsky, to appear in "Correlated Fermions and Transport in Mesoscopic Systems", ed. by G. Montambeaux and T. Martin.

[16] B. Pannetier, J. Chaussy, and R. Rammal, Phys. Scripta T 13, 245-251, (1986).

[17] S. G. den Hartog, C. M. A. Kapteyn, B. J. van Wees, and T. M. Klapwijk, Phys. Rev. Lett. 76, 4592 (1996). 